\title{
Strain inhomogeneity in copper islands probed by coherent $x$-ray diffraction
}

G. Beutier, M. Verdier, G. Parry, B. Gilles, S. Vu Hoang, P.-F. Lory, M. de Boissieu, F. Livet SIMaP, 1130 rue de la piscine, BP 75, Saint-Martin d'Hères cedex, France

\author{
S. Labat, M.-I. Richard, O. Thomas
}

Aix-Marseille University, CNRS, IM2NP, FST, avenue Escadrille-Normandie-Niemen, 13397

Marseille cedex, France

\section{T. Cornelius}

European Synchrotron Radiation Facility, 6 rue Jules Horowitz, BP 220, 38043 Grenoble cedex, France

\begin{abstract}
The strain field of individual epitaxial sub-micrometric copper islands is studied using coherent x-ray diffraction and finite element modelling. The strain inhomogeneity in each island is so large that the characteristic features of the island shape tend to disappear in the diffraction pattern, which is dominated by strain effects. The model confirms the tensile strain imposed to the island by the thermal mismatch occurring during the preparation of the samples. An evaluation of the residual strain is obtained by qualitatively fitting the diffraction data.
\end{abstract}

\section{Keywords}

strain field, nanostructures, copper, coherent x-ray diffraction

\section{Introduction}

There is a great interest in the study of size effects on mechanical properties, driven by the technological importance of isolated nanostructures such as wires or islands. The general observation that 'smaller is stronger' [1] still lacks of good understanding of the underlying physical mechanisms. In crystalline materials, the characterisation of the microstructure (strain field and defects) is of crucial importance to understand the mechanical response (see for example [2]). Coherent X-ray Diffraction (CXD) is a non-destructive technique used to investigate the 3D displacement field of an object [3]. The focus of this work concerns the CXD investigation of a model system of metallic islands: 
sub-micrometric crystals of $\mathrm{Cu}$ are obtained by solid state dewetting of a very thin $\mathrm{Cu}$ film on a Ta (001) single crystal, avoiding any alloying or damage, for example due to Focus Ion Beam machining of lithographic steps. $\mathrm{Cu}$ is chosen as a model material since it is prone to plastic deformation at room temperature and the physics of its plastic deformation is well known. The broad scope of our study is to observe in situ defects nucleation in such crystals by contact mechanical loading (nanoindentation). In the present paper we focus on the as-grown state of the islands.

We report the preparation route for such crystals and detail the experimental CXD results in the first two sections. To evaluate three-dimensional displacement fields, two strategies can be used: either by using a model in direct space, for example with a Finite Element Method (FEM), from which the diffraction is computed and compared to the experimental observation, or by inverting a reciprocal space map, using iterative algorithms and oversampled data to compensate for the loss of the phase in intensity measurements. In the third section we analyse and discuss our results of the as-deposited crystals using the former strategy.

\section{Sample preparation and lab characterization}

$\mathrm{Cu}$ on Ta substrate is a unique system concerning solid state dewetting. On a Ta (001) surface, nanothin films of $\mathrm{Cu}$ are metastable and dewet into $\mathrm{Cu}$ crystallites at high temperature [4,5]. A high purity Ta crystal, cut and polished to less than $0.1^{\circ}$ along the (001) plane, was purchased from Goodfellow. The surface is prepared in ultra-high vacuum environment by repeated Ar sputtering and flash annealing above $2400 \mathrm{~K}$ [6], until Low-Energy Electron Diffraction shows that the surface is atomically flat. A 5 $\mathrm{nm}$ thick film of $\mathrm{Cu}$ is then deposited at room temperature and dewetted by heating at $1200 \mathrm{~K}$ for 3 minutes. Figure 1 shows typical dewetted $\mathrm{Cu}$ crystallites. Their shape is close to the equilibrium state, with (001) facets on the top and (111) facets dominating on the sides. The typical dimensions of the islands studied in this article are $800 \times 800 \mathrm{~nm}^{2}$ with height ranging from 100 to $400 \mathrm{~nm}$ as measured by Atomic Force Microscopy. Their unique crystallographic orientation with respect to the substrate,

shown by the perfect alignment of the squared shapes (Figure 1), is $\mathrm{Cu}(001) / / \mathrm{Ta}(001)$ and $\mathrm{Cu}[110] / / \mathrm{Ta}[100]$, as determined by Electron Backscatter Diffraction.

\section{HERE FIGURE 1}

Figure 1: Secondary Electron Microscopy image of the dewetted copper islands. The enlargement in insight shows the crystallographic axes. 
Laboratory X-ray diffraction measurements confirm the orientation relationship and show that the out-of-plane lattice parameter of the copper islands is $0.65 \%$ smaller than the nominal bulk value for copper. Moreover, the islands are very inhomogeneously strained, which is reflected in the large broadening of their diffraction peaks, that can not be attributed to a size effect: the typical Full Width at Half Maximum (FWHM) of rocking curves on the $\mathrm{Cu} 002$ Bragg reflection is $0.3^{\circ}$, as compared to $0.05^{\circ}$ for the substrate 002 Bragg reflection (with a copper source). This large residual strain in the islands probably results from the difference $\Delta \alpha=\alpha_{\mathrm{Ta}}-\alpha_{\mathrm{Cu}}$ of thermal expansion coefficients $\alpha$ between Ta and $\mathrm{Cu}$ : the cooling down of $\Delta \mathrm{T} \approx-800^{\circ} \mathrm{C}$ from the dewetting temperature to room temperature would indeed lead to $\Delta \alpha \Delta \mathrm{T} \approx 0.82 \%$ of residual tensile strain if the deposition is considered as a continuous thin film $\left(\alpha_{\mathrm{Ta}}=6.310^{-6} \mathrm{~K}^{-1}\right.$ and $\left.\alpha_{\mathrm{Cu}}=16.510^{-6} \mathrm{~K}^{-1}\right)$.

\section{Coherent x-ray diffraction}

CXD measurements are performed at beamline ID01 of the ESRF with a monochromatic X-ray beam of $8 \mathrm{keV}$. The set-up to ensure coherence and sub-micron focusing is detailed in [7]. The profile of the beam at the sample position is measured by scanning a thin wire of tantalum. Its size (FWHM $\approx 0.6 \times 0.3$ $\left.\mu \mathrm{m}^{2}, \mathrm{HxV}\right)$ is in qualitative agreement with values reported in [7]. Its footprint on the sample for an incidence corresponding to the $\mathrm{Cu} 002$ Bragg angle $\left(\sim 25.4^{\circ}\right)$ allows measuring the 002 Bragg reflection of individual islands in symmetrical geometry. The scattered intensity is recorded with a $256 \times 256$ pixels Maxipix detector (ESRF) mounted at $0.88 \mathrm{~m}$ from the sample. We investigated a large number of copper islands and recorded three-dimensional maps of the reflection by rocking the sample over $\sim 1^{\circ}$ [8]. The diffraction patterns of the copper islands look all very similar. A typical one is shown in Figure 2. Isosurfaces of the diffraction patterns appear as square-based pyramids with strong rounded edges and fringes on their faces. The fringes are nearly perpendicular to the $\mathrm{z}$ axis, but they are bent by the large strain inhomogeneity. The 002 Bragg reflection of Tantalum is far enough $\left(\sim 2.5^{\circ}\right.$ higher in Bragg angle) so that it has no effect in the measured region of the reciprocal space. The Crystal Truncation Rod of the substrate surface traverses the $\mathrm{Cu} 002$ reflection, creating strong interferences. The dispersion of sizes and aspect ratios (length/width/height) of the islands is somehow reflected in the diffraction patterns, but without significant effect on the main features, and the global shape is conserved. It is clear that the large strain inhomogeneity is dominant in the diffraction pattern, such that no size and shape effect can be easily evidenced. 
Figure 2: 002 Bragg reflection of a typical $\mathrm{Cu}$ island. (a): as seen on the detector at the maximum of the rocking curve. (b): three-dimensional map reconstructed from the rocking curve. An isosurface is shown. (c): (qx, qz) slice through the peak. (d): (qx, qy) slice through the peak (it is truncated at the bottom by the too short range of the rocking curve).

\section{Modelling and discussion}

In principle, the shape, density and strain field of the diffracting crystal can be reconstructed from the oversampled diffraction pattern only [9], but in practice the method still remains difficult, particularly in the case of highly strained crystals [10]. Here we use a different approach, which is modelling the object to simulate its diffraction pattern and compare it to experimental data [11].

The deformation of a typical island with base $1 \mu \mathrm{m} \times 1 \mu \mathrm{m}$ and height $400 \mathrm{~nm}$ is modelled together with the substrate using FEM. The strain field is calculated assuming only thermoelastic deformations (no plasticity), simulating the differential contraction of the lattice parameters as the system is cooled down by $800^{\circ} \mathrm{C}$, i.e. $\Delta \alpha \Delta \mathrm{T}=0.8 \%$. The elastic anisotropy of both materials and their respective crystallographic orientation are taken into account. The equilibrium state after cooling is used to calculate the diffraction pattern. With this purely elastic model, we can arbitrarily rescale the strain field without rerunning the FEM calculations. The displacement field, relevant to the calculation of the diffracted intensity, is readily obtained from the strain field by integration.

The diffracted intensity of a strained object can be expressed as [12]:

$$
I(\vec{q}) \propto\left|\int \rho(\vec{r}) e^{\vec{q} \cdot \vec{r} \cdot \vec{r}} d \vec{r}\right|^{2} \approx \mid F T\left[e^{i \vec{Q} \cdot \vec{u}(\vec{r})}\right]^{2}
$$

where $r$ is the running variable in space, $\rho(r)$ the electronic density, taken constant in the island and

null outside, $u(r)$ the displacement field, $q$ the general scattering vector and $Q$ the scattering vector at the Bragg peak. We note that only the component of the displacement field along the diffraction vector $Q$ impacts on the diffraction pattern: for the 002 Bragg reflection, only the displacement field along $z$ matters. Here the integration is performed using the Fast Fourier Transform (FFT), after a linear interpolation of the sampling points from the FEM mesh. The approximation in equation (1) holds for constant $\rho(r) \equiv 1$ and $|(q-Q) \cdot u(r)| \ll 2 \pi$. Although this second assumption is largely wrong in the 
present case for some combinations of $q$ and $r$, the formula still gives a reasonable result, allowing an easy computation and a qualitative comparison with experimental data.

Indeed, the calculations presented in Figure 3, obtained with an elastic field such that the average compression of the island along $z$ was $\varepsilon_{z z}=-0.65 \%$, reproduce remarkably well the general shape of the experimental diffraction pattern.

\section{HERE FIGURE 3}

Figure 3: $(a):(x, z)$ slice through the copper island showing the displacement field $u_{z}$ (colourscale in nanometres) for $\Delta \alpha \Delta T=0.4 \%\left(\varepsilon_{z z}=-0.65 \%\right)$. (b): isosurface (1\% of the maximum value) of the Cu 002 Bragg reflection computed from the same displacement field.

While most CXD studies reported in the literature deal with weakly strained objects, such that the features of the diffraction pattern are mostly related to the shape, the strain in the system studied here is so inhomogeneous that the shape has no obvious impact on the diffraction pattern. To better understand how the internal strain alters the diffraction pattern, we varied the thermal mismatch $\Delta \alpha \Delta \mathrm{T}$ in our simulations. Sample results are shown in Figure 4.

\section{HERE FIGURE 4}

Figure 4: (qx,qz) slices of the simulated 002 copper Bragg reflection for various values of the thermal mismatch. The corresponding value $\varepsilon_{z z}$ of the average deformation of the island along $z$ is also given. (a): $\Delta \alpha \Delta T=-0.4 \%\left(\varepsilon_{z z}=0.65 \%\right),(b): \Delta \alpha \Delta T=0\left(\varepsilon_{z z}=0\right),(c): \Delta \alpha \Delta T=0.1 \%\left(\varepsilon_{z z}=-0.16 \%\right),(d): \Delta \alpha \Delta T=0.2 \%$ $\left(\varepsilon_{z z}=-0.32 \%\right),(e): \Delta \alpha \Delta T=0.4 \%\left(\varepsilon_{z z}=-0.65 \%\right),(f): \Delta \alpha \Delta T=0.8 \%\left(\varepsilon_{z z}=-1.3 \%\right)$.

The effect of the shape is given by the case $\Delta \alpha \Delta \mathrm{T}=0$ : the fringes on the vertical streak are due to interferences between top and bottom sides, and the inclined streaks result from the 111 facets. These features are altered as soon as inhomogeneous strain is introduced, and rapidly disappear. In particular, the strain is responsible for the loss of the symmetry. Inverting the sign of the thermal mismatch (and thus of the strain field) inverts the diffraction pattern. It is clear that the experimental data correspond to the case of tensile strain in the island $(\Delta \alpha \Delta \mathrm{T}>0)$. The case $\Delta \alpha \Delta \mathrm{T}=0.1 \%$ is the order of magnitude expected in 'free' standing metal particles on a support. The case $\Delta \alpha \Delta \mathrm{T}=0.8 \%$ corresponds to the case in which the system behaves like a thin film on the substrate. A qualitatively good agreement with the 
experimental data is obtained for about half this value, i.e. $\Delta \alpha \Delta \mathrm{T} \sim 0.4 \%$. This value gives an average deformation along the $z$ axis of $\varepsilon_{\mathrm{zz}}=-0.65 \%$, also in good agreement with the experimental value obtained from the position of the Bragg reflection only. We conclude that the system relaxes partially during the cooling, possibly by generating dislocations or a wetting layer at the interface.

\section{Conclusions}

We have studied the strain field in $\mathrm{Cu}$ sub-micrometric islands dewetted on a Ta (001) substrate, by CXD and FEM. The residual strain field in the islands is large and so inhomogeneous that it dominates the diffraction pattern, and characteristic features related to the shape tend to disappear. A systematic investigation of many islands shows that the dispersion of size and shape do not play a great role and that the strain field is very similar from one island to the other. By simulating the CXD patterns with the strain field obtained from FEM, we are able to reproduce the general shape and characteristic features of diffraction patterns. The residual strain giving the best qualitative agreement with the experimental data corresponds to about half the residual strain that would be expected in a continuous thin film. The partial strain relaxation is possibly due to the creation of dislocations or a wetting layer at the interface. This study confirms that CXD, combined with FEM [11], can still be very useful to characterize highly strained crystals, a case in which direct algorithmic imaging of the strain field remains problematic.

\section{References}

1. H. D. Espinosa, S. Berbenni, M. Panico, and K. W. Schwarz, Proc. Natl. Acad. Sci. USA 102 (2005) 16933.

2. H. Bei, S. Shim, G.M. Pharr, E.P. George, Acta Mater. 56 (2008) 4762 .

3. Ian Robinson and Ross Harder, Nature Mater. 8 (2009) 291.

4. L. Chen, N. Magtoto, B. Ekstrom, and J. Kelber, Thin Solid Films 376 (2000) 115.

5. V. Venugopal, $\mathrm{PhD}$ Thesis, Defects and thermal stability of nanothin $\mathrm{Cu}$ films on Mo and $\mathrm{Ta}$, Technische Universiteit Delft 2005.

6. H. Yamazaki, K. Sakamoto, A. Fujii, T. Kamisawa, Surf. Sci. 563 (2004) 41-47.

7. F. Mastroprietro, D. Carbone, A. Diaz, J. Eymery, A. Sentenac, T. H. Metzger, V. Chamard, and V. Favre-Nicolin, Opt. Express 19 (2011) 19223.

8. G. J. Williams, M. A. Pfeifer, I. A. Vartanyants, and I. K. Robinson, Phys. Rev. Lett. 90 (2003) 
175501.

9. Mark A. Pfeifer, Garth J. Williams, Ivan A. Vartanyants, Ross Harder \& Ian K. Robinson, Nature 442 (2006) 63.

10. A. A. Minkevitch, M. Gailhanou, J.-S. Micha, B. Charlet, V. Chamard, and O. Thomas, Phys. Rev. B 76 (2007) 104106.

11. Ana Diaz, Virginie Chamard, Cristian Mocuta, Rogerio Magalhães-Paniago, Julian Stangl, Dina Carbone, Till H Metzger and Günther Bauer, New J. Phys. 12 (2010) 035006.

12. V Favre-Nicolin, F Mastropietro, J Eymery, D Camacho, Y M Niquet, B M Borg, M E Messing, L-E Wernersson, R E Algra, E P A M Bakkers, T H Metzger, R Harder and I K Robinson, New J. Phys. 12 (2010) 035013. 